\title{
MANIFESTACIONES OFTALMOLÓGICAS EN TUMORES CEREBRALES PEDIÁTRICOS: 58 CASOS
}

\section{OPHTHALMOLOGICAL FINDINGS IN PEDIATRIC BRAIN NEOPLASMS: 58 CASES}

\author{
SANTAMARÍA A ${ }^{1}$, MARTÍNEZ R ${ }^{2}$, ASTIGARRAGA I ${ }^{2}$, ETXEBARRÍA J ${ }^{2}$, SÁNCHEZ M ${ }^{1}$
}

\section{RESUMEN}

Objetivos: Describir las manifestaciones visuales en neoplasias neuropediátricas, relacionarlas con la localización tumoral y analizar su relevancia en el seguimiento de la enfermedad.

Material y métodos: Estudio descriptivo retrospectivo que incluye pacientes menores de 14 años con neoplasias cerebrales de cualquier estirpe, registradas en nuestro centro desde 1996 hasta el 2005.

Resultados: En un 44\% de los pacientes con descenso en la agudeza visual, la ambliopía fue catalogada como orgánica. En el $28 \%$ de los casos, la ambliopía era secundaria al estrabismo/nistagmus producido por el tumor. El tratamiento corrector resultó efectivo en varios casos de ambliopía parcial y totalmente orgánica. Tres recidivas tumorales fueron detectadas gracias a la exploración de fondo de ojo y campimetría, antes de que las pruebas de imagen fueran concluyentes.

Conclusión: El examen oftalmológico (agudeza visual, campimetría, funduscopia) es una pieza clave en el seguimiento de la patología tumoral cerebral. Las ambliopías orgánicas parciales/totales son susceptibles de tratamiento corrector.

Palabras clave: Tumores cerebrales, ambliopía, campimetría, agudeza visual, astrocitoma.

\begin{abstract}
Purposes: To describe the visual manifestations of brain neoplasms, and to analyze the effect of tumor control on these.

Methods: This is a descriptive retrospective study, which includes patients under 14 years of age, suffering from different brain neoplasms in our hospital between 1996 and 2005 inclusive.

Results: In the group of patients with low visual acuity, $44 \%$ had organic amblyopias. In $28 \%$ of cases, the amblyopia was secondary to the strabismus/nystagmus produced by the developing tumor. Corrective treatment was successful in some cases of partial or total organic amblyopia. Ophthalmologic evaluation (including perimetry and fundoscopy) enabled detection of 3 tumor relapses.

Conclusions: Visual analysis (visual acuity, perimetry, fundoscopy) should be an essential component of assessment during brain neoplasm treatment and follow-up. Corrective treatment is sometimes successful in organic amblyopias (Arch Soc Esp Oftalmol 2008; 83: 471-478).
\end{abstract}

Key words: Brain neoplasms, amblyopia, perimetry, visual acuity, astrocytoma.

\footnotetext{
Recibido: 28/5/07. Aceptado: 26/6/08.

Servicios de Oftalmología y Pediatría del Hospital de Cruces. Barakaldo (España).

1 Licenciado en Medicina.

2 Doctor en Medicina.

Comunicación presentada en el Congreso de la Sociedad Oftalmológica del Norte de España (SONE) (Pamplona, 2006).
}

Correspondencia:

Alaitz Santamaría Carro

Cl. Ganguren, $6,5 .^{\circ} \mathrm{B}$

48530 Ortuella (Vizcaya)

España

E-mail: alaitz_sc@yahoo.es 


\section{INTRODUCCIÓN}

El ojo representa una ventana abierta al sistema nervioso central, y como tal, puede proporcionarnos múltiples y variados signos como resultado de una alteración a cualquier nivel de la vía óptica y/o de las numerosas estructuras asociadas que toman parte en el complejo proceso de la visión. En la siguiente revisión de casos pretendemos describir y analizar las diferentes manifestaciones oftalmológicas producidas por procesos neoplásicos intracraneales en una población pediátrica determinada. Clasificaremos esta información y la interpretaremos como un componente más en una disfunción de carácter severo y con potencial afectación multiorgánica.

\section{SUJETOS, MATERIAL Y MÉTODO}

Con intención de estudiar las manifestaciones oftalmológicas de los tumores cerebrales en un subgrupo concreto de población pediátrica, se planteó un estudio descriptivo retrospectivo que abarcaba todos los procesos neoplásicos cerebrales de cualquier estirpe (incluyendo las metástasis intracraneales de tumores con foco primario a distancia) presentadas en niños menores de 14 años en nuestro centro. En el estudio se incluyeron los pacientes controlados a lo largo de los últimos diez años, basándonos en las fechas de alta registradas entre 1996 y 2005 (ambos incluidos). Los objetivos del estudio fueron los siguientes:

- Revisar y clasificar los tumores cerebrales pediátricos atendidos en nuestro centro desde 1996 hasta 2005.

- Estudiar de los signos y síntomas oftalmológicos en cada paciente.

- Estudiar la relación de la localización tumoral con la clínica oftalmológica.

- Interpretar las pruebas complementarias oftalmológicas.

- Valorar el papel de la exploración oftalmológica en cuanto al diagnóstico y seguimiento de la enfermedad.

- Analizar los diferentes tratamientos oftalmológicos empleados y su respuesta en cada caso.

Para la recopilación de datos se recurrió al siguiente material: Historias clínicas disponibles en los archivos del hospital; historias escaneadas en el sistema informático por fallecimiento del paciente $\mathrm{u}$ otros motivos no especificados; historias remitidas al servicio de oftalmología o a otras áreas hospitalarias (oncología pediátrica, neuropediatría, ortopedia infantil, endocrinología) por hallarse en activo en el momento del estudio. Posteriormente se procedió a la recogida de datos, que se clasificaron en una cuartilla patrón con los siguientes apartados: Datos identificativos del paciente; patología cerebral, especificando tipo de tumor, localización y extensión, edad de presentación y número de recaídas en su caso; patologías secundarias al proceso tumoral o al tratamiento recibido; clínica visual (agudeza visual subjetiva/objetiva, ambliopías y corrección en cada caso, hallazgos en la inspección ocular/biomicroscopía/fondo de ojo, trastornos del nervio óptico, trastornos de otros pares craneales y otras alteraciones en la motilidad ocular); pruebas complementarias oftalmológicas (campimetrías, potenciales evocados visuales, test de colores, test de diplopía, etc.); pruebas de imagen representativas (resonancia magnética cerebral y tomografía axial computerizada cerebral); tratamiento oftalmológico/general recibido durante el período de estudio; éxitus en su caso, fecha de defunción y edad del paciente.

\section{RESULTADOS}

Tras la recopilación de los datos a estudiar, se realizó el análisis y clasificación de la información en los siguientes apartados: Estirpe tumoral; agudeza visual/ametropías; disfunción de pares craneales; nistagmus; hallazgos en biomicroscopía/fondo de ojo; campimetría; éxitus.

\section{Estirpe tumoral (tabla I, fig. 1)}

Los tumores más frecuentes entre todos los revisados pertenecieron a la estirpe neuroglial. En este grupo se detectaron 13 astrocitomas, 3 de ellos supraselares, 4 de fosa posterior (cerebelosos), 4 hemisféricos de fosa anterior, un astrocitoma de células gigantes del tercer ventrículo y uno pilocítico medular cervical. Entre los gliomas distinguimos 3 troncoencefálicos, 2 del nervio óptico/quiasma, uno talámico y una gliomatosis cerebri difusa en un síndrome de Neurofibromatosis tipo I. Para finalizar con este grupo citamos 5 tumores neurogliales derivados del epéndimo. 
Tabla I. Manifestaciones oftalmológicas según la estirpe tumoral

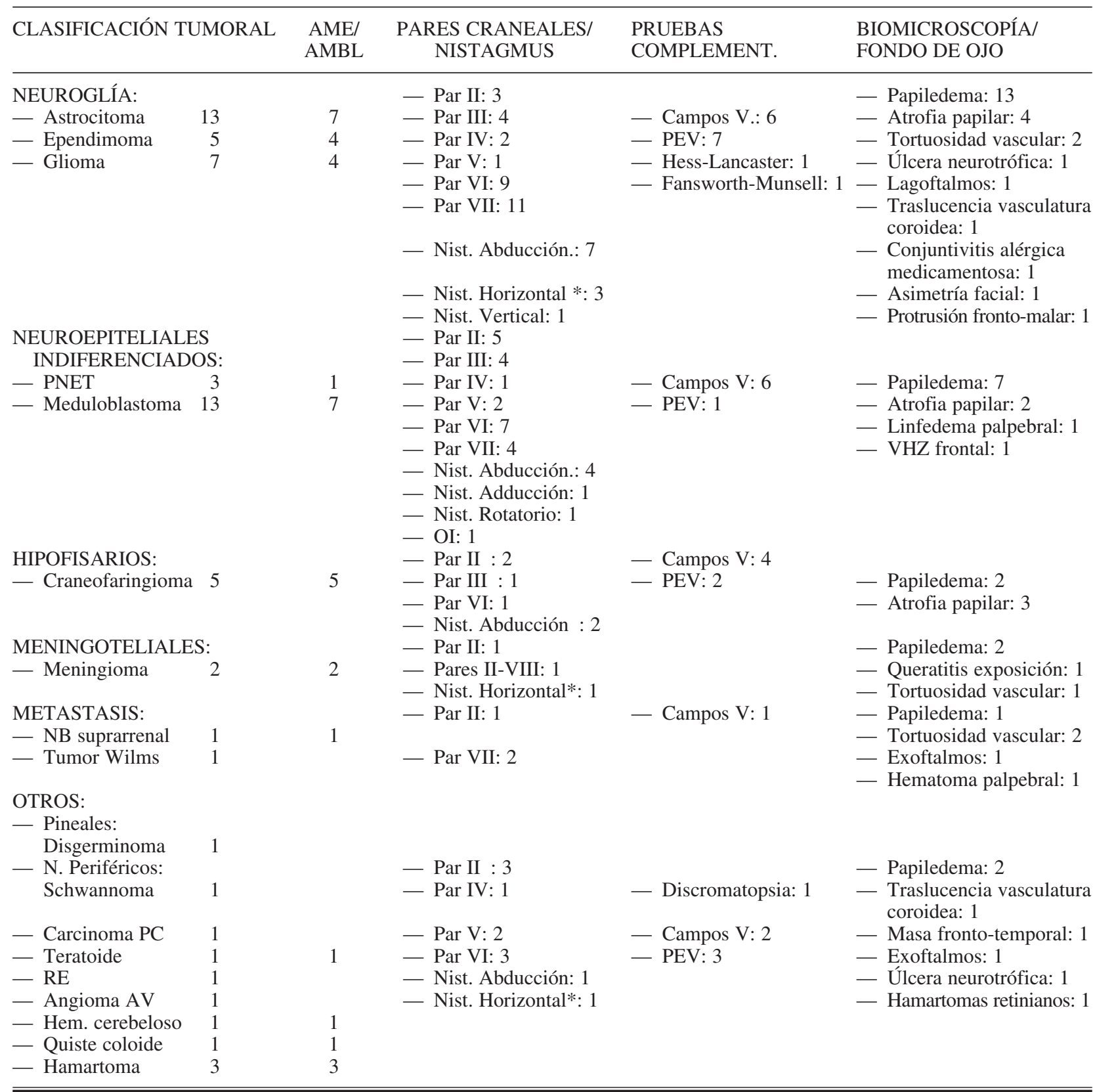

* Nistagmus horizontal no especificado.

AME/AMBL: ametropías/ambliopías; Campos V: campos visuales; PEV: potenciales evocados visuales; PNET: tumores derivados del neuroectodermo primitivo; VHZ: virus herpes zoster; OI: oftalmoplejía internuclear; NB: neuroblastoma; RE: rabdomiosarcoma embrionario; Angioma AV: angioma arteriovenoso; Hem. cerebeloso: hemangioblastoma cerebeloso; Carcinoma PC: carcinoma de plexos coroideos.

Los segundos tumores en frecuencia fueron los neuroepiteliales indiferenciados, entre los que destacamos los tumores hemisféricos derivados del neuroectodermo primitivo (PNET) (3 casos) y los meduloblastomas del vérmix cerebeloso, que repre- sentaron el grupo individual más numeroso con 13 casos registrados.

Los tumores hipofisarios hallados en la población pediátrica a estudio fueron exclusivamente craneofaringiomas (5 casos). 


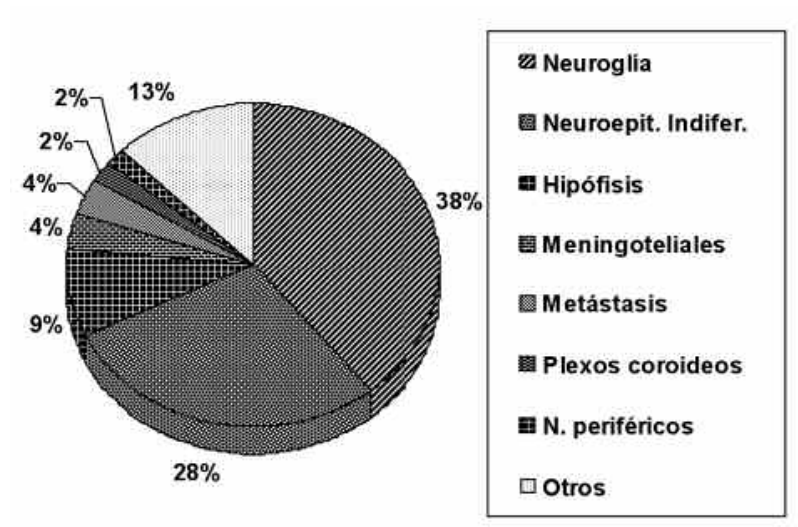

Fig. 1: Clasificación de los tumores neuropediátricos según su estirpe.

Dentro de la estirpe meningotelial se hallaron 2 casos de meningioma; refiriéndonos a los tumores de nervios periféricos, se detectó un tumor de las vainas nerviosas (schwannoma). Sendos procesos correspondieron a pacientes afectos de neurofibromatosis tipo 2. Los 3 casos de hamartomatosis cerebral descritos pertenecían a dos pacientes con esclerosis tuberosa y uno con neurofibromatosis tipo 1.

Dos de las neoplasias cerebrales estudiadas resultaron ser metástasis de focos primarios a distancia (tumor renal de Wilms y neuroblastoma suprarrenal). Del mismo modo, 10 tumores entre los citados produjeron diseminación metastática leptomeníngea. Seis de ellos fueron meduloblastomas cerebelosos, que se perfilan como una de las estirpes de peor pronóstico.

Entre todas las neoplasias clasificadas, un $42 \%$ tenían origen en la fosa craneal posterior.

\section{Agudeza visual/ametropías}

El registro de la agudeza visual se llevó a cabo únicamente en 36 pacientes, entre los cuales se determinó una pérdida de visión tanto subjetiva como objetiva en el $77 \%$ de los casos. Tras analizar detenidamente la etiopatogenia del déficit visual (fig. 2), se concluyó que en un $44 \%$ de los casos existía una alteración orgánica visual que justificaba el trastorno, y que estaba producida directamente por el proceso tumoral, o secundariamente a su expansión, a la distorsión de las estructuras adyacentes o al aumento de presión intracraneal generado. Sin embargo, existe un grupo paralelo (28\%) en el que el tumor produjo una reducción indirecta de

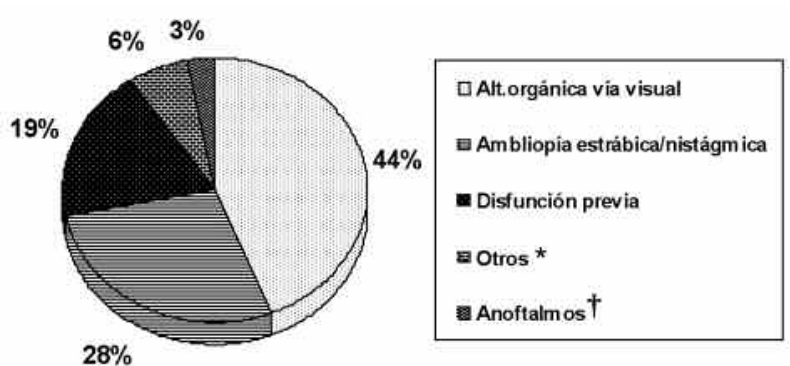

$28 \%$

Fig. 2: Etiopatogenia de la disminución de la agudeza visual en el grupo de estudio.

* Se incluye un caso de autoestimulación ocular/fotofobia en un astrocitoma del tercer ventrículo, y un frotamiento ocular persistente en un meduloblastoma diseminado. †Anoftalmos posquirúrgico en un paciente con meningioma del nervio óptico afecto de neurofibromatosis tipo 1.

la agudeza visual. Nos referimos a las ambliopías estrábicas o nistágmicas producidas por el daño en los núcleos-pares oculomotores como consecuencia del avance de la masa intracraneal. Podríamos sin duda incluir estos sujetos en el apartado «Ambliopías orgánicas o secundarias al proceso tumoral» ya que se trata de secuelas indirectas del tumor, pero decidimos estudiarlas como un grupo independiente por su especificidad y por la peculiaridad de su fisiopatología.

En un $19 \%$ de los sujetos se halló un trastorno previo en la agudeza visual, predominando las ambliopías por estrabismos convergentes acomodativos en sujetos hipermétropes. Denominaremos a este grupo «Disfunciones previas» o «Ambliopías no orgánicas», y señalamos que en todas ellas se obtuvo una ganancia en la agudeza visual tras la corrección adecuada en cada caso (gafas; oclusiones; penalizaciones; inyecciones de toxina botulínica; cirugía de estrabismo). Más aún, detectamos una mejoría visual notable al administrar corrección a varios pacientes con un componente ambliópico orgánico parcial (asociado a un trastorno visual previo al desarrollo tumoral) o exclusivamente orgánico (fig. 3).

\section{Pares craneales}

En nuestro estudio consideramos el segundo par craneal o nervio óptico como un grupo independiente por la gran variedad de signos/síntomas que 


\section{AVI AMETROPÍAS}

- Caso 1: Astrocitoma pilocitico cerebeloso

- Esotropía acomodativa

- OD: $20 / 40$

- OI: 20120

GAFAS + OCLUSION

$20 / 20$

$20 / 20$

- Caso 2:CFG

- Esotropía acomodativa

- Atrofia papilar 6/6; PEV patológicos OD; CV alterados $A O$

- OD: $20 / 200$

$20 / 25$

- Ol: $20 / 25$

GAFAS + OCLUSION

$20 / 20$

- Caso 3: Glioma quiasma óptico (ORGANICIDAD)

- OD: $20 / 50$

- OI: $20 / 200$

GAFAS + OCLUSIÓN

$20 / 50$

$20 / 70$

Fig. 3: Ejemplo de mejoría en la agudeza visual tras corrección en pacientes con ambliopía previa al desarrollo tumoral, ambliopía combinada (previa y orgánica) y ambliopía exclusivamente orgánica.

OD: ojo derecho; OI: ojo izquierdo; AO: ambos ojos; PEV: potenciales evocados visuales; $C V$ : campos visuales.

puede generar. Entre las alteraciones más características destacamos las siguientes: Alteraciones pupilares (18 casos); discromatopsia (4 casos); amaurosis autolimitada (3 casos); ceguera legal (agudeza visual menor de 0,1) en 3 casos y anoftalmos secundario a exenteración en un meningioma del nervio óptico. En cuanto al aspecto del disco óptico en la funduscopia, 17 pacientes presentaron papiledema en algún momento del proceso, y en 14 de ellos se objetivó una atrofia papilar residual. Se registraron tres casos en los que el papiledema fue el primer dato indicativo de recidiva tumoral en una fase en la que la RMN y el resto de pruebas complementarias aún no eran resolutivas.

Refiriéndonos al resto de pares craneales, y como podemos observar en la figura 4, el más frecuentemente afectado fue el abducens (VI) por su gran susceptibilidad a las compresiones y a las variaciones en la presión intracraneal (1). El segundo par en frecuencia fue el facial (VII). Este hecho puede explicarse por la proximidad de ambos pares craneales tanto en su salida del tronco del encéfalo como en su trayecto intracraneal (2). Les sigue el par III con un $18 \%$, y el IV, V y VII en menor frecuencia.

\section{Nistagmus (fig. 5)}

En la población a estudio, el tipo más frecuente de nistagmus es el de componente horizontal que se

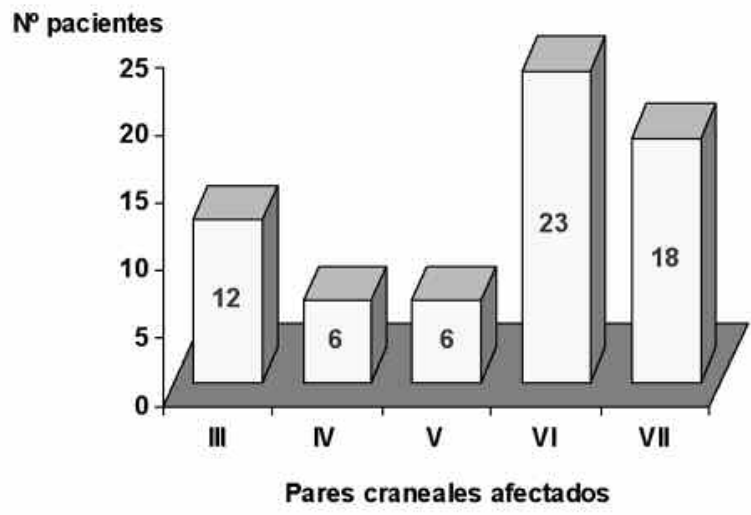

Fig. 4: Frecuencia de parálisis/paresias de pares craneales.

exacerba en las lateroversiones. En un $61 \%$ hallamos un nistagmus horizontal bilateral, y en el $24 \%$ de los casos fue unilateral. Este hecho es explicable por la gran frecuencia de afectación del sexto par en los procesos neoplásicos intracraneales, limitando la abducción de uno o ambos ojos y generando un nistagmus secundario. Los nistagmus verticales aparecen en una proporción notablemente menor.

\section{Biomicroscopía/fondo de ojo}

Son numerosos y variados los hallazgos en la inspección ocular, biomicroscopía y fondo de ojo. Como hemos comentado anteriormente, el edema de papila y la atrofia papilar son habituales en el grupo de estudio. El papiledema suele presentarse como uno de los signos precoces de la enfermedad, como consecuencia de una compresión directa del nervio óptico y/o un aumento en la presión intracraneal que compromete el retorno venoso ocular. Por lo general, el papiledema y los síntomas de hipertensión intracraneal se resuelven tras la

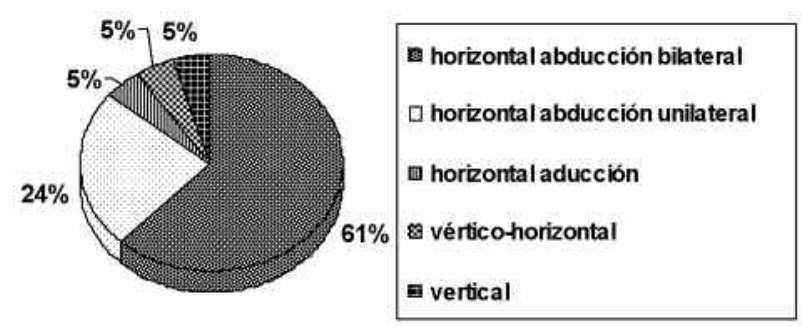

Fig. 5: Clasificación de los nistagmus en el grupo de estudio. 
implantación de una válvula de derivación ventrículo-peritoneal. La atrofia papilar, que se caracteriza por palidez y por un aumento en la excavación, suele ser consecutiva al edema de papila y por lo tanto aparece en fases más tardías (1). El estudio incluye otras múltiples manifestaciones, detalladas en la tabla I.

\section{Campimetría (figs. 6-9)}

A lo largo del estudio encontramos diversos patrones campimétricos específicos según la localización tumoral (3), y que son de gran utilidad no sólo en el diagnóstico y localización anatómica de la neoplasia, sino también en el seguimiento de la enfermedad y en la detección de recidivas. Refiriéndonos a dos casos en particular, fue posible realizar un diagnóstico precoz de una recidiva tumoral gracias a la modificación de los campos visuales de los pacientes y a la reciente aparición de una diplopía binocular en uno de ellos.

\section{Éxitus}

El número de pacientes fallecidos ascendió a 20 (34\%); 5 pacientes con edades comprendidas entre los 0 y 4 años, 5 pacientes entre 5 y 9 años, y 10 pacientes entre 10 y 15 años, con edad media de fallecimiento de 6,16 años. Un $25 \%$ de los casos corresponde a meduloblastomas del vérmix cerebeloso. En la mayoría de los casos $(60 \%)$ no se especifica la causa de la muerte, catalogándose como parada cardiorrespiratoria. Un $20 \%$ de los niños

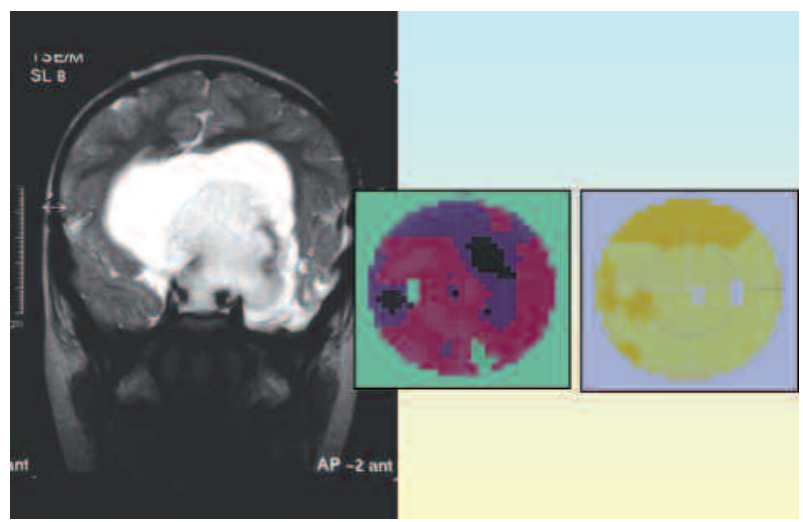

Fig. 6: Campimetría I: anopsia izquierda en un astrocitoma pilocítico supraselar con hidrocefalia obstructiva.
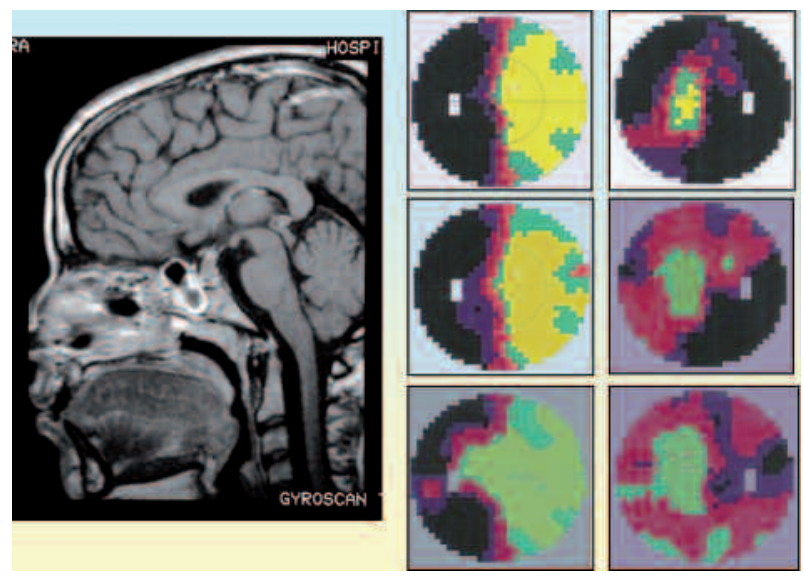

Fig. 7: Campimetría II: Evolución campimétrica de un craneofaringioma. Obsérvese la discreta mejoría en los sucesivos estudios.

sufrieron una insuficiencia respiratoria objetivable. En el 20\% restante incluimos una insuficiencia cardíaca, una hemorragia cerebral masiva, un shock séptico y un estatus convulsivo.

\section{DISCUSIÓN}

Las neoplasias sólidas más frecuentes en la edad pediátrica son las correspondientes al sistema nervioso central. Las estirpes tumorales predominantes varían respecto al adulto, siendo más comunes en
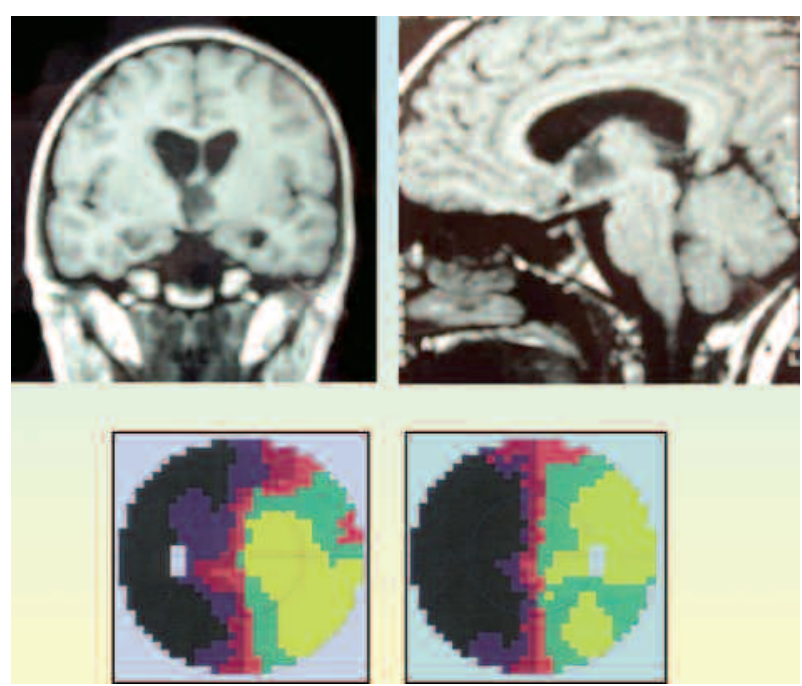

Fig. 8: Campimetría III: hemianopsia homónima izquierda en un quiste coloide del tercer ventrículo con afectación de la cintilla óptica derecha. 


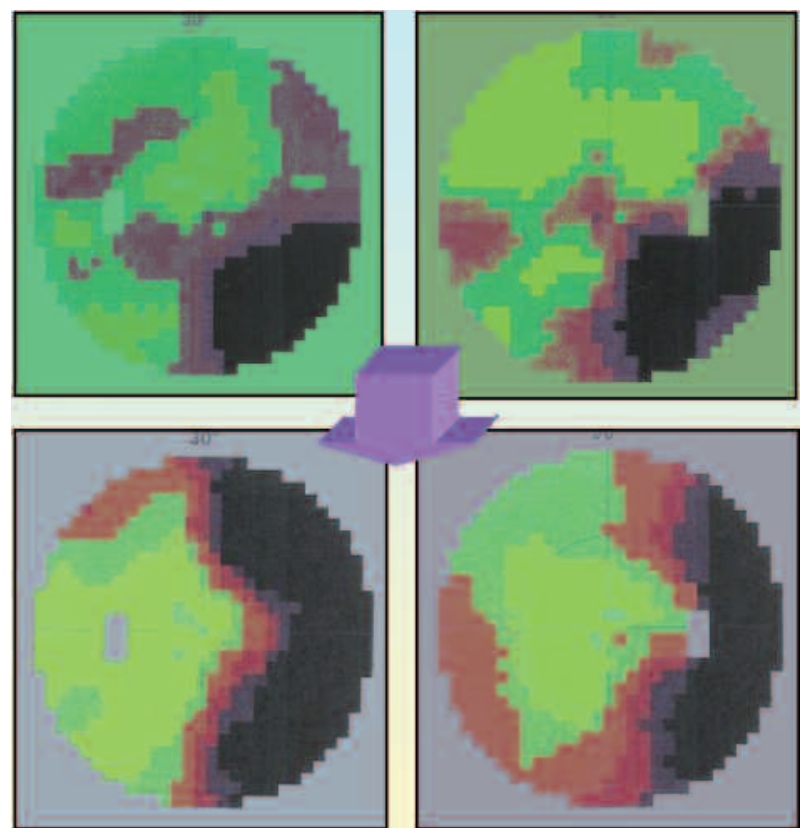

Fig. 9: Campimetría IV: Cuadrantanopsia inferior derecha en un ependimoma parietal izquierdo. Obsérvese la progresión a hemianopsia derecha como resultado de la extensión tumoral hacia el lóbulo temporal izquierdo.

niños los gliomas benignos, tumores neuroepiteliales indiferenciados y craneofaringiomas $(4,5)$. Los meduloblastomas del vérmix cerebeloso representan el grupo individual más numeroso (6), dato que concuerda con los resultados de nuestro estudio. En cuanto a la localización tumoral, el porcentaje de neoplasias infantiles supratentoriales es equiparable a las infratentoriales, a diferencia del adulto, en cuyo caso predominan las primeras $(4,7)$.

Como hemos comprobado, la exploración oftalmológica es de vital importancia para diagnosticar recidivas de tumores cerebrales pediátricos en los que no existen otras manifestaciones neurológicas y las pruebas de imagen no son aún concluyentes. En nuestra serie fue posible detectar precozmente varias recidivas tumorales gracias al hallazgo de papiledema en la exploración funduscópica de rutina (3 casos), y a las variaciones recientes en la cam- pimetría ( 2 casos). La recidiva tumoral es la causa más frecuente de muerte tardía en pacientes pediátricos. Por lo tanto, el pronóstico mejora si se detecta la recurrencia en fase asintomática (4). Según nuestro criterio, una exploración oftalmológica óptima básica en pacientes neuropediátricos debería incluir: agudeza visual espontánea/con corrección y esquiascopia; examen de la motilidad ocular/estudio estrabismo; biomicroscopía y fondo de ojo; campimetría; otros (test de diplopía, test de binocularidad/supresión, etc.).

Refiriéndonos a las alteraciones en la agudeza visual, hemos comprobado que la corrección de las ambliopías no orgánicas («disfunciones previas») presenta resultados similares a los obtenidos en la población sana. Más aún, las ambliopías por lesión orgánica directa o producidas por un estrabismo/nistagmo secundario al tumor son potencialmente corregibles. Por todo ello, no creemos conveniente subestimar la capacidad de rehabilitación visual del niño oncológico, ya que en ocasiones la respuesta al tratamiento es inesperada y es posible obtener un nivel visual aceptable para su manejo en la vida cotidiana.

\section{BIBLIOGRAFÍA}

1. Kanski JJ. Oftalmología clínica. V Ed. Madrid: Elsevier; 2004.

2. Putz R, Pabst R. Atlas de anatomía humana Sobotta. XX Ed. Madrid: Panamericana; 1997; I: 266.

3. Martin JH. Neuroanatomía. II Ed. Hertfordshire: Prentice Hall; 1998; 190-193.

4. Pollack IF. Pediatric brain tumors. Semin Surg Oncol 1999; 16: 73-90.

5. Sklar CA. Childhood brain tumors. J Pediatr Endocrinol Metab 2002; 15: 669-673.

6. Rickert $\mathrm{CH}$, Paulus W. Epidemiology of central nervous system tumors in childhood and adolescence based on the new WHO classification. Childs Nerv Syst 2001; 17: 503511.

7. Mehta V, Chapman A, McNeely PD, Walling S, Howes WJ. Latency between symptom onset and diagnosis of pediatric brain tumors: an Eastern Canadian geographic study. Neurosurgery 2002; 51: 365-373. 